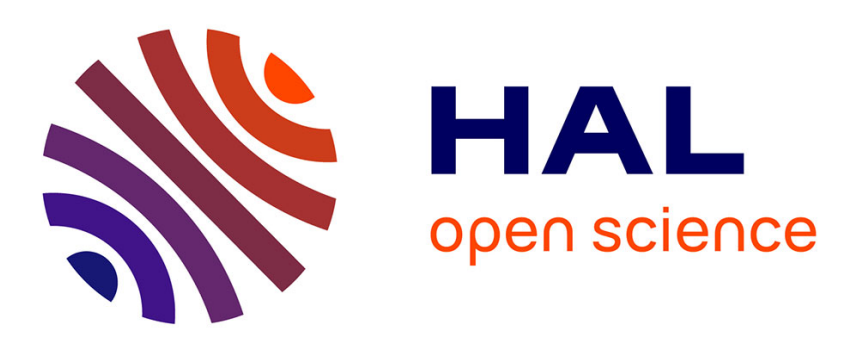

\title{
Anomalous density dependence of structural relaxation time in water
}

Filippo Bencivenga, Andrea Cimatoribus, Alessandro Gessini, Maria Grazia

Izzo, Claudio Masciovecchio

\section{To cite this version:}

Filippo Bencivenga, Andrea Cimatoribus, Alessandro Gessini, Maria Grazia Izzo, Claudio Masciovecchio. Anomalous density dependence of structural relaxation time in water. Philosophical Magazine, 2008, 88 (33-35), pp.4137-4142. 10.1080/14786430802302067 . hal-00513929

\section{HAL Id: hal-00513929 \\ https://hal.science/hal-00513929}

Submitted on 1 Sep 2010

HAL is a multi-disciplinary open access archive for the deposit and dissemination of scientific research documents, whether they are published or not. The documents may come from teaching and research institutions in France or abroad, or from public or private research centers.
L'archive ouverte pluridisciplinaire HAL, est destinée au dépôt et à la diffusion de documents scientifiques de niveau recherche, publiés ou non, émanant des établissements d'enseignement et de recherche français ou étrangers, des laboratoires publics ou privés. 


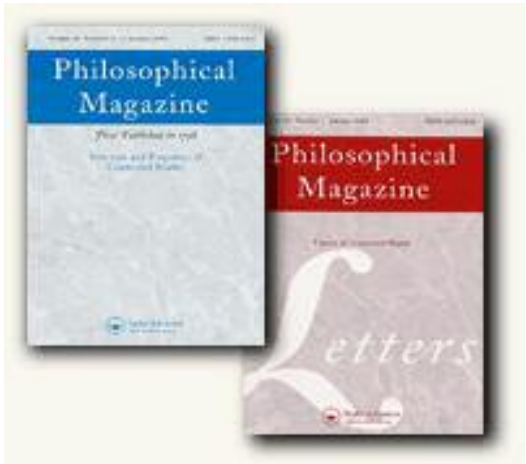

Anomalous density dependence of structural relaxation time in water

\begin{tabular}{|r|l|}
\hline Journal: & Philosophical Magazine \& Philosophical Magazine Letters \\
\hline Manuscript ID: & TPHM-08-May-0161.R1 \\
\hline Journal Selection: & Philosophical Magazine \\
\hline Author: & 04-Jun-2008 \\
\hline Complete List of Authors: & $\begin{array}{l}\text { Bencivenga, Filippo; ESRF } \\
\text { Cimatoribus, Andrea; ELETTRA } \\
\text { Gessini, Alessandro; ELETTRA } \\
\text { Izzo, Maria; ELETTRA } \\
\text { Masciovecchio, Claudio; ELETTRA }\end{array}$ \\
\hline Keywords (user supplied): & inelastic scattering \\
\hline fluids, high-pressure effects \\
\hline $\begin{array}{l}\text { Note: The following files were submitted by the author for peer review, but cannot be converted } \\
\text { to PDF. You must view these files (e.g. movies) online. }\end{array}$ \\
\hline Wat_HP_2.tex \\
\hline
\end{tabular}

\section{S) ScholarONE" \\ Manuscript Central}




\title{
Anomalous density dependence of structural
} relaxation time in water

\author{
Filippo Bencivenga*†, Andrea Cimatoribus ${ }^{\dagger}$, \\ Alessandro Gessini ${ }^{\dagger}$, Maria Grazia Izzo ${ }^{\dagger \dagger}$, Claudio Masciovecchio ${ }^{\dagger \S}$
}

4th June 2008

\begin{abstract}
The density dependence of water structural relaxation time has been determined by inelastic ultraviolet scattering (IUVS). The observed trend is opposite with respect to the expected one. This striking experimental result calls for further investigations.
\end{abstract}

\section{Introduction}

Water occupies a central role in liquid state physics both because it is the most important liquid for everyday life and because understanding the origin of its several anomalies is a challenge for physicists [1]. Among various aspects, the viscoelastic behavior of water has been and still is being the subject of several experimental works $[2,3,4,5,6,7]$. As a common result the presence of a relaxation process with a characteristic time scale, $\tau \approx p s$, was observed. One of the most spectacular effects of such a relaxation is a modification of the characteristic frequency of longitudinal sound propagation, $\Omega_{L}$, that, according to classical hydrodynamics, must be equal to $c_{s} Q$, where $c_{s}$ and $Q$ are the adiabatic sound velocity and the wavevector, respectively. At low frequency, i.e., when the condition $\Omega_{L} \ll \tau^{-1}$ is met (viscous regime), the hydrodynamic expectations are verified. On the other hand, as far as $\Omega_{L} \approx \tau^{-1}$, an increase of $\Omega_{L}$ with respect to $c_{s} Q$ is clearly observed. Finally, for $\Omega_{L} \gg \tau^{-1}$ the degrees of freedom responsible for the relaxation process are indeed too slow in order to efficiently dissipate the energy carried by the acoustic wave, which therefore propagates without energy losses. In such an elastic regime the sound propagation frequency asymptotically reaches a high frequency limit, $\Omega_{L}=$ $c_{\infty} Q$, where $c_{\infty}>c_{s}$ is usually referred to as infinite frequency sound velocity.

\footnotetext{
*filippo.bencivenga@elettra.trieste.it

${ }^{\dagger}$ Sincrotrone Trieste, 34012 Basovizza, Trieste, Italy

${ }^{\ddagger}$ Dipartimento di Fisica, Universitá degli Studi di Trieste, 34127 Trieste, Italy

${ }^{\S}$ CRS SOFT-INFM-CNR, at Universitá di Roma La Sapienza, 00185 Roma, Italy
} 
This viscoelastic phenomenology allows probing the internal dynamics of the fluids by monitoring their effects on sound wave propagation $[8,9]$.

The advent of novel hypersonic techniques, such as inelastic x-ray (IXS) and ultraviolet (IUVS) scattering, opened up the possibility to directly determine sound propagation frequencies in the $T H z$ range and, therefore, to detect relaxation processes with $p s$ and sub-ps timescales. In recent years IXS experiments pointed out that the temperature $(T)$ dependence of $\tau$ follows an activation (Arrhenius) behavior whit an activation energy similar to the hydrogen bond (HB) one $[4,5]$. This result strongly suggests that the underlying physical origin of the relaxation is the collective rearrangements of the local structure after a perturbation (such as an acoustic wave) through the making and breaking of HB. Finally, recent IUVS results on supercooled liquid water at room pressure highlight a good consistency with the predictions of Mode Coupling Theory (MCT) [6]. This latter result has relevant implications in understanding the strongly debated origin of water anomalies. In fact MCT is one of the so-called singularity free scenario theories that, together with the liquid-liquid phase transition hypothesis, are the most promising frameworks for describing water behavior [1].

However, the density $(\rho)$ dependence of $\tau$ was only poorly investigated up to now. In particular, there are not any systematic studies accomplished in the low $T$ region of water phase diagram, where most of anomalies show up. We then decided to exploit IUVS technique in order to investigate the $\rho$ behavior of $\tau$. The choice of IUVS was motivated by the twofold reasons: i) IUVS has proven to be able to determine $\tau$ in the $T$-range of interest. ii) The throughput of the employed IUVS spectrometer is high enough in order to allow the reliable accomplishment of a systematic study.

\section{Experimental method}

The experiment was performed at BL10.2 at ELETTRA light laboratory (Trieste, Italy). The spectrometer is based on a Czerny-Turner optical scheme able to provide an overall frequency resolution of $\Delta \omega / \omega \approx 10^{-6}$. Moreover, a position sensitive detector allows one to measure the inelastic signal, $I(Q, \omega)$, at a given scattering angle, $\theta_{S}=172^{\circ}$, in one single shot, thus avoiding timeconsuming monochromator scans. Since the $T$ and $\rho$ dependencies of water refraction index $(n)$ is known in the probed range [10], the probed $Q$ can be straightforwardly determined:

$$
Q=2 n \omega_{i} \sin (\theta / 2) / c
$$

where $\mathrm{c}$ is the speed of light in vacuum and $\omega_{i}$ is the frequency of the incoming radiation ( $7.710^{15} \mathrm{~Hz}$ in the present case).

The sample was embedded in a high pressure hydrostatic cell (HPHC) made of Inconel 625 with $3 \mathrm{~mm}$ thick sapphire optical windows. The cell was connected to an external pressure generator through a capillary. The pressure $(P)$ stability was better than \pm 5 bar. The HPHC was kept in thermal contact with a thermal 
sink and an electronically controlled resistive heater, providing an overall $T$ stability better than $0.2 \mathrm{~K}$. The sample density at a given $(P, T)$-value was calculated from water Equation of State (EoS) [11].

Fig. 1 displays some selected IUVS spectra of water at $T=273 K, Q \approx 0.07$ $\mathrm{nm}^{-1}$ at the indicated $\rho$-values. The symmetric inelastic side features are clearly evident, as well as a large central peak ( $>90 \%$ of the whole spectral intensity). This mainly arises from spurious reflections by the HPLC windows. $I(Q, \omega)$ is thus proportional to the dynamical structure factor, $S(Q, \omega)$, convoluted with the instrumental resolution function, $R(\omega)$, plus the spurious elastic component, i.e.:

$$
I(Q, \omega)=\left[A_{1} S(Q, \omega)+A_{2} \delta(\omega)\right] \bigotimes R(\omega)+B,
$$

where $A_{1}$ and $A_{2}$ are weighting factors, $\otimes$ is the convolution integral and $B$ is a flat background mainly due to the detector dark count. $S(Q, \omega)$ was modeled by a damped harmonic oscillator (DHO) function:

$$
S(Q, \omega)=\pi^{-1} \frac{\Omega_{L}^{2} \Gamma_{L}}{\left[\omega^{2}-\Omega_{L}^{2}\right]^{2}+\left[\omega \Gamma_{L}\right]^{2}},
$$

where $\Gamma_{L}$ is the damping coefficient of the longitudinal sound modes. A best fit procedure based on a standard $\chi^{2}$ minimization allowed extracting the values of both $\Omega_{L}$ and $\Gamma_{L}$. Particularly good $(<1 \%)$ was the accuracy in the determination of $\Omega_{L}$. For the forthcoming discussion it is worth stressing that, analytically, $\Omega_{L}$ represents the frequency at which the longitudinal current spectrum, $J(Q, \omega)=\omega^{2} S(Q, \omega) / Q^{2}$, assumes its maximum value. This is not a mere coincidence since, formally, the characteristic frequency of the longitudinal sound modes corresponds to the maxima of $J(Q, \omega)$ instead of the ones of $S(Q, \omega)[12]$.

\section{Data analysis}

The usual way for extracting $\tau$ from $S(Q, \omega)$ is to use an appropriate model function where the relaxation parameters appear explicitly. Among various possible choices the memory function formalism has proven to be very well suited for a wide range of system $[4,5,6,12,13,14,15]$. In this framework $S(Q, \omega)$ reads:

$$
\frac{S(Q, \omega)}{S(Q)}=\frac{1}{\pi} \frac{\left(c_{s} Q\right)^{2} m^{\prime}(Q, \omega)}{\left[\omega^{2}-\left(c_{s} Q\right)^{2}-\omega m^{\prime \prime}(Q, \omega)\right]^{2}+\omega^{2}\left[m^{\prime}(Q, \omega)\right]^{2}},
$$

where $m^{\prime}(Q, \omega)$ and $m^{\prime \prime}(Q, \omega)$ are the real and imaginary parts of the Fourier transform of the memory function that, in a first approximation, can be written as a single exponential time decay [12]:

$$
m(Q, t)=\left(c_{\infty}^{2}-c_{s}^{2}\right) Q^{2} e^{-t c_{s}^{2} / \tau c_{\infty}^{2}}
$$


Indeed, the above ansatz is largely used and it was found to very well describe the dynamical response of several non conductive liquids, including water $[4,5$, 12, 13]. By imposing the condition

$$
\left(\frac{\partial\left(\omega^{2} S(Q, \omega)\right)}{\partial \omega}\right)_{\omega=\Omega_{L}}=0,
$$

whit $S(Q, \omega)$ given by Eqs. 4 and 5 , it is possible to obtain an analytical relation that links $\tau$ with $c_{s}, c_{\infty}$ and $\Omega_{L}$ :

$$
\tau=\left(c_{\infty} / c_{s}\right)^{2} \sqrt{\frac{1-R^{-2}}{2 c_{\infty}^{2} Q^{2}-2 \Omega_{L}^{2}}},
$$

where $R=\left(\Omega_{L} / c_{s} Q\right)^{2}$. The experimentally observed density behavior of $R$ is reported in the left panel of Fig. 2. We can notice that IUVS can accurately determine very small deviation of $\Omega_{L}$ with respect to $c_{s} Q$. Finally, since the $\rho$-dependence of $c_{\infty}$ can be extrapolated by previous IXS works $[4,16,17]$ and $c_{s}$ can be accurately calculated from the EoS [11] (see Fig. 2), the experimental determined values of $\Omega_{L}$ can be used for calculating $\tau$ through Eq. 7 .

\section{Results and discussion}

The obtained results are reported in Fig. 3. A clear monotonic $\rho$-decrease of structural relaxation time in the probed density range is observed. This trend is inconsistent with the one expected by considering simple free volume arguments. In fact, the free volume reduction at high density would in principle lead to a $\rho$-increase of $\tau$ that, at very high density, may turn into divergence when pressure induced vitrification occurs. A qualitative example of such an expected trend is reported in Fig. 3 as full line [18]. It is worth remarking that the obtained results do not strongly depend on the parameter $c_{\infty}$ that, indeed, is the one with the lower experimental accuracy. In fact, by keeping $c_{\infty}$ fixed at two extremes (i.e., 3800 and $2500 \mathrm{~m} / \mathrm{s}$ ), only small quantitative discrepancies were found, while the overall trend is preserved (see Fig. 3).

In conclusion, we determined the density dependence of relaxation time by using the IUVS technique. We clearly found an unexpected $\rho$-dependence of $\tau$ which cannot be accounted for any existing theories. Such a preliminary result clearly deserves further investigations.

\section{References}

[1] O. Mishima and H.E. Stanley The relationship between liquid, supercooled and glassy water, Nature 396 (1998), 329.

[2] C. M. Davis and J. Jarzynski, Water: a Comprehensive Treatise, Vol. 1, edited by F. Franks, Plenum Press, New York (1972). 
[3] A. Cunsolo and M. Nardone, Velocity dispersion and viscous relaxation in supercooled water, J. Chem. Phys. 105 (1996), 3911.

[4] G. Monaco et al., Viscoelastic behavior of water in the terahertz-frequency range: An inelastic x-ray scattering study, Phys. Rev. E 60 (1999), 5505.

[5] F. Bencivenga et al., High-frequency dynamics of liquid and supercritical water, Phys. Rev. E 75 (2007), 051202.

[6] C. Masciovecchio et al., Structural Relaxation in LiquidWater by Inelastic UV Scattering, Phys. Rev. Lett. 92 (2004), 255507.

[7] S.C. Santucci et al., Is there any fast sound in water?, Phys. Rev. Lett. 97 (2006), 225701.

[8] J. Frenkel and J. Obraztsov, , J. Exptl. Theor. Phys. URSS 9 (1939), 1081.

[9] J. Markham, R.T. Beyer and R.B. Linsday, Absorption of Sound in Fluids, Rev. Mod. Phys. 23 (1951), 353.

[10] P. Schiebener et al., Refractive Index of Water and Steam as Function of Wavelength, Temperature and Density, J. Phys. Chem. Ref. Data 19 (1990), 677.

[11] W. Wagner and A. Pruss, The IAPWS Formulation 1995 for the Thermodynamic Properties of Ordinary Water Substance for General and Scientific Use, J. Phys. Chem. Ref. Data 31 (2002), 387.

[12] J.P. Boon and S. Yip, Molecular Hydrodynamics, McGraw-Hill International Book Company, New York (1980).

[13] F. Bencivenga et al., Structural and Collisional Relaxations in Liquids and Supercritical Fluids, Phys. Rev. Lett. 98 (2007), 085501.

[14] T. Scopigno, G. Ruocco and F. Sette, Microscopic dynamics in liquid metals: The experimental point of view, Rev. Mod. Phys. 77 (2005), 881.

[15] R. Angelini et al., Microscopic dynamics and relaxation processes in liquid hydrogen fluoride, Phys. Rev. B 70 (2004), 224302.

[16] M. Krisch et al., Pressure Evolution of the High-Frequency Sound Velocity in LiquidWater, Phys. Rev. Lett. 89 (2002), 125502.

[17] In Fig. 2 we report $c_{\infty}$ values corresponding at $\mathrm{T}=277$ and $273 \mathrm{~K}$ (from $[4,16])$.

[18] P.B. Macedo and T.A. Litovitz, On the Relative Roles of Free Volume and Activation Energy in the Viscosity of Liquids, J. Chem. Phys. 42 (1965), 245. The line shown in Fig. 3 corresponds to Eq. 10 where $\gamma=0.75$ and $v_{0}=0.0115 \mathrm{~nm}^{3}$. 
Figure 1: Selection of IUVS spectra of water at $273 \mathrm{~K}, Q \approx 0.07 \mathrm{~nm}^{-1}$ at the indicated $\rho$-values.

Figure 2: Left panel: experimental values of $\mathrm{R}$ (see text for further details). Right panel: density dependence of $c_{\infty}$ (open dots, $[4,16,17]$ ) and $c_{s}$ (full lines, [11])

Figure 3: Density dependence of $\tau$ as obtained by using Eq. 7 (see text for further details). The open up (down) triangles are the results obtained by keeping $c_{\infty}=3800(2500) \mathrm{ms}$. The full line qualitatively depicts the expected trend [18]. 


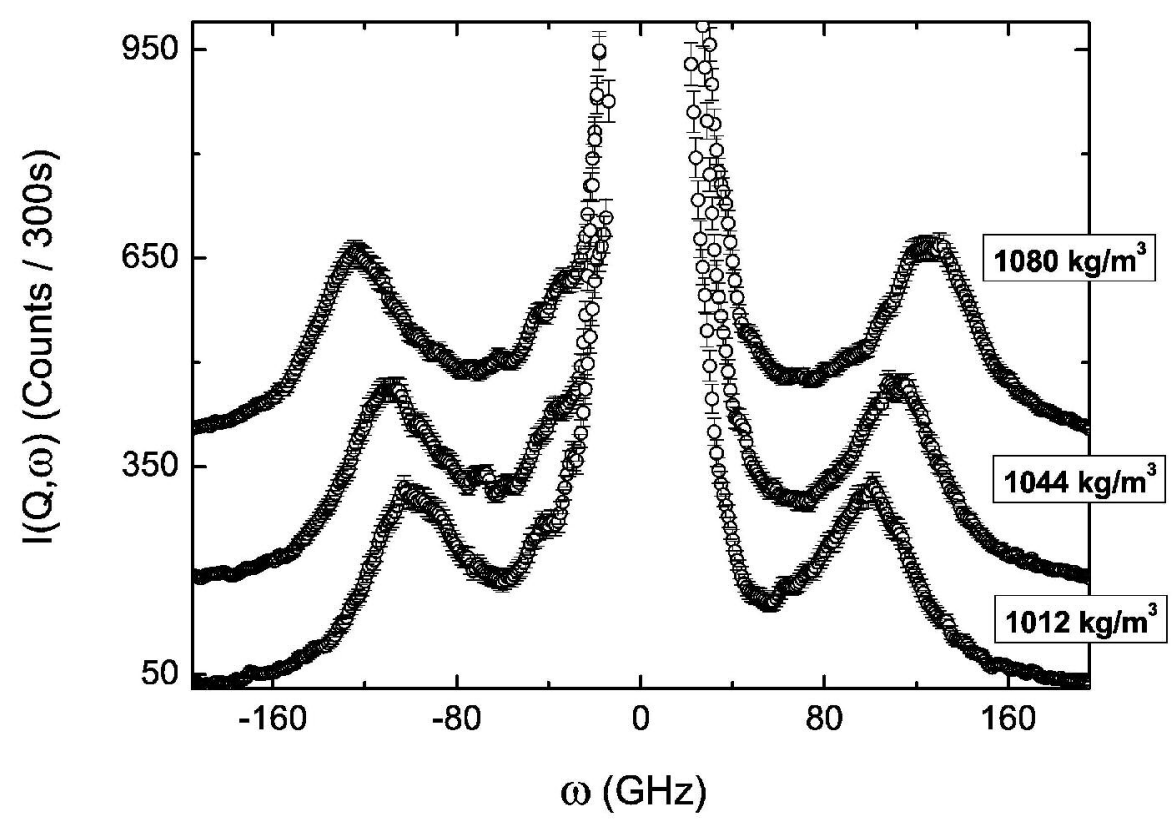

$117 \times 89 m m(600 \times 600$ DPI $)$ 

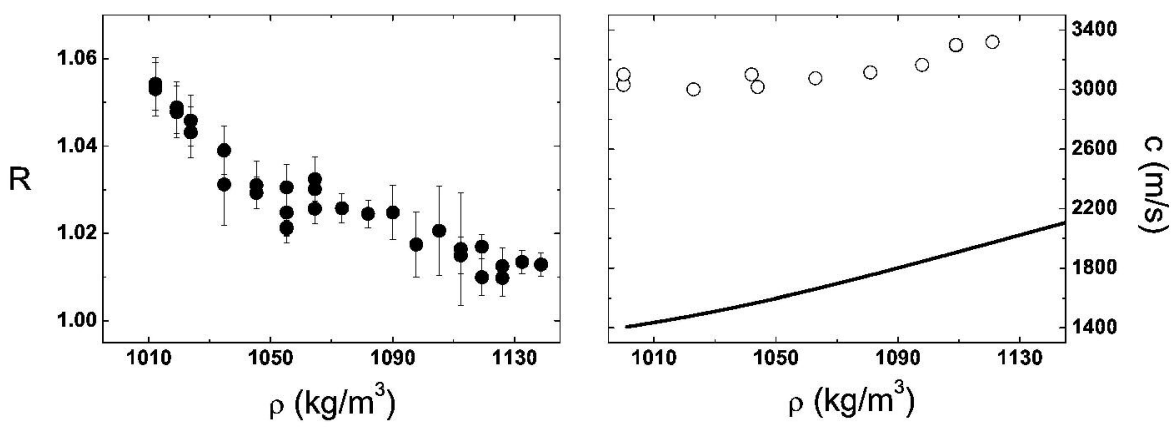

$197 \times 84 \mathrm{~mm}(600 \times 600 \mathrm{DPI})$ 
Page9osfo:hical Magazine \& Philosophical Magazine Letters

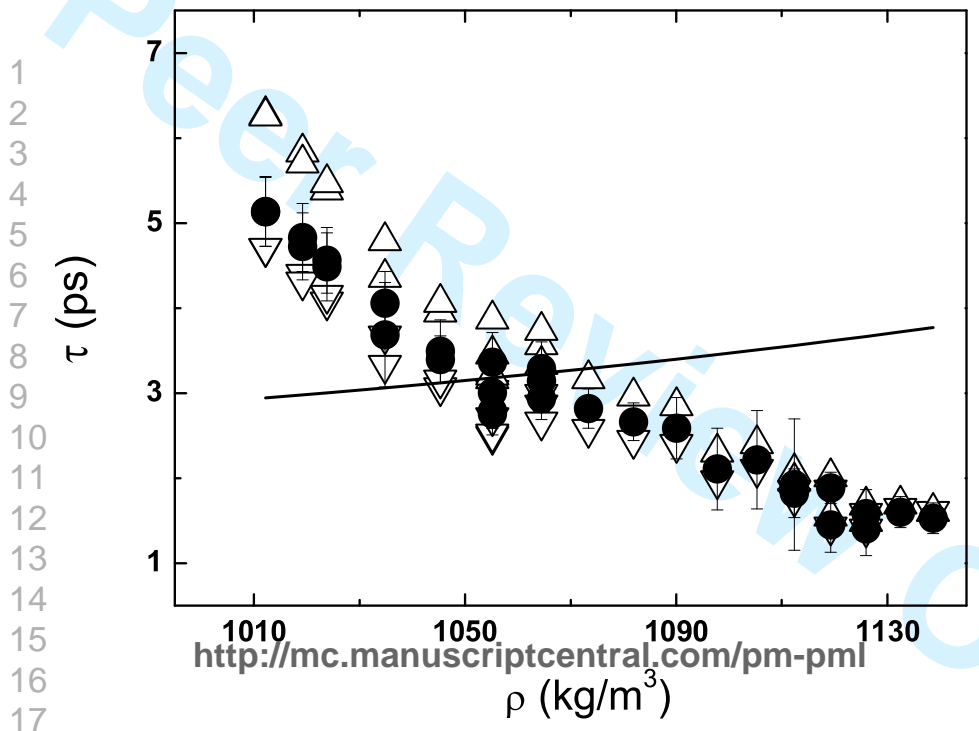

